\title{
MODEL OF $\mathrm{HfO}_{2} / \mathrm{Al}_{2} \mathrm{O}_{3}$ DIELECTRIC AIN InN\AIGaNMOS HEMT FOR POWER APPLICATION
}

\author{
N. Anbuselvan, P. Anandan, A. Manju
}

\begin{abstract}
The work involves to devise dielectric $\mathrm{HfO}_{2} / \mathrm{Al}_{2} \mathrm{O}_{3} \mathrm{MOS}-\mathrm{HEMT}$ stack to validate for the device structure through the refinement of mesh analysis. The structure of the AlNVInN $\backslash A I G a N$ HEMT MOS-HEMTs are designed and meshed using the script file in the Sentaurus structure editor. TheAlNVInNAIGaN MOS-HEMTs output characteristic are examined for physics of MOS-HEMT in Hydrodynamic model TCAD. The amalgamation formation ofthe $A l_{0.30} G a N_{0.70}$ barrierinterface with the channelofhigh $2 D E G$ density result in anincreaseddrain current density and high trans conductance. Additional the device performance of DC transfer characteristics, small signal analysis of AlN/InN/AlGaNMOS-HEMT are analyzed and simulated.
\end{abstract}

Keywords: MOS-HEMT,AlNVInNALGaN, Hydrodynamic model, $A l_{0.30} G a N_{0.70}$ and2DEG.

\section{INTRODUCTION}

The built in composite semiconductor HEMTs devise remains to be widely used for the application of the devices with lower-noise immune, higher voltage, and power-switching characteristics of the microwave/ millimeter-wave Integrated Circuit ([1-2]. Nevertheless, the gate leakage challenges in thermionic release at barrier of a Schottky-gate due to elevation electric fields and surface charger at the barrier of gate-drain regional behavior would remarkably reduce the device performance. There are varies types of gate dielectric materials have be employed in the MOS-gate device to improve the gate insulated driving capability. By accounting varies dielectric impact in MOS-HEMT devices along with different gate dielectric material are studied by including many processing varying parameter in the performances too be deliberate in future $\mathrm{Ga}$ based devices. This work given as better insight to the MOS-HEMTs with different compound $\mathrm{HfO}_{2} / \mathrm{Al}_{2} \mathrm{O}_{3}$ dielectric layers to designed for power application in $\mathrm{RF}$ regime [1-10].

The cost operative ozone water oxidization technique to device fabrication achieved at room temperature below the atmospheric pressure disadvantaged in the exclusive deposition system. Hence an interfacial distinction and diminished surface states defects are over meowing to presence of the barrier over the GaN layer with respected to earlier said method of process. In particularly the gate-channel separation with respected to the charges

Revised Manuscript Received on November 10, 2019.

N.Anbuselvan, Assistant Professor, Department of EEE, Saveetha School of Engineering, Thandalam, Chennai.

P.Anandan, Professor, Department of ECE, C.Abdul Hakeem College of Engineering and Technology, Melvisharam.

A.Manju, Professor, Department of EEE, Saveetha School of Engineering, Thandalam, Chennai. concentration in the oxide layer are made to oxidization and deposited to it. Moreover, to recover the gate leakage current a competence methods where included along with the equivalent oxide thickness i.e. $\mathrm{HfO}_{2}$ of higher dielectric constant $(\mathrm{k})$ in additional to the deposition of the $\mathrm{Al}_{2} \mathrm{O}_{3}$ layer through Radio frequency sputtering method. The foremost feature of the MOS-HEMT is its higher carrier mobility and polarization related to the positive impact on the current of the device. Hence by inducting these simulating parameter of the high mobility and polarization effects, the physical model of the device are validated in sentaurus bundle.

The classical transport models of the Drift-Diffusion (DD) and the Hydro-Dynamic (HD) model cannot be directly employed for conventional ways of simulating physics of the devices in quantum well. Hence in order simulationAIN\InN\AlGaNHEMT devices, the Hydrodynamic Density Gradient model (HDG) is altered by considerations of following set equations governed by the basic Poisson equation, the electron current continuity equation, the quantum effects equation, carrier impact ionizations effects, scattering mechanism and energy band gap balance equation besides with induced polarization effects. Then these interrelated parameter of distinction and dielectric property are categorized by means of low-frequency analysis and capacitance voltage analysis duly owing to change in characteristics of DC and Radio Frequency parameters are investigated in this work.

\section{METHODOLOGY}

The models proposed here be able to straightforwardly be implemented in TCAD simulation bundles, these models for $\mathrm{GaN}$ devices are using.

1. The TCAD simulations of AlN $\backslash$ InN $\backslash A 1 G a N t o$ precisely match restrained small signal AC statistics.

2. To diminish the DC gate leakage currents and to avert RF current collapse.

3. To reveal TCAD AC analysis and RF simulations.

4. To recover the breakdown voltages and RF parameter control value.

5. To influence the technique for sinking on-resistance with the dielectrics of high dielectric constant beside device varies device dimensions.

The device modeled structure consists of theAlN/InNbuffer layer on a silicon substrate, with an extremely resistive nature of carbon dopingofGaN layer of a thickness around $2.0 \mu \mathrm{m}$, along undoped thicknessGaN layer of $100 \mathrm{~nm}$ and the $\mathrm{Al}_{0.30} \mathrm{GaN}_{0.70}$ barrier of abstaining $25 \mathrm{~nm}$ of thickness. In order to confirmation deeper acceptor higher resistivity nature of GaN: $\mathrm{C}$ layer at $0.9 \mathrm{eV}$ [7], ashallow donor quanta of concentration charges of 
$1 \times 10^{15} \mathrm{~cm}^{-3}$ is doped in the energy level of valence band and traps density for all nitrides based layers [8].The depth in $\mathrm{Al}_{0.30} \mathrm{GaN}_{0.70}$ barrier layer beneath gate and gate dielectric thickness of $25 \mathrm{~nm}$ and $40 \mathrm{~nm}$, respectively.

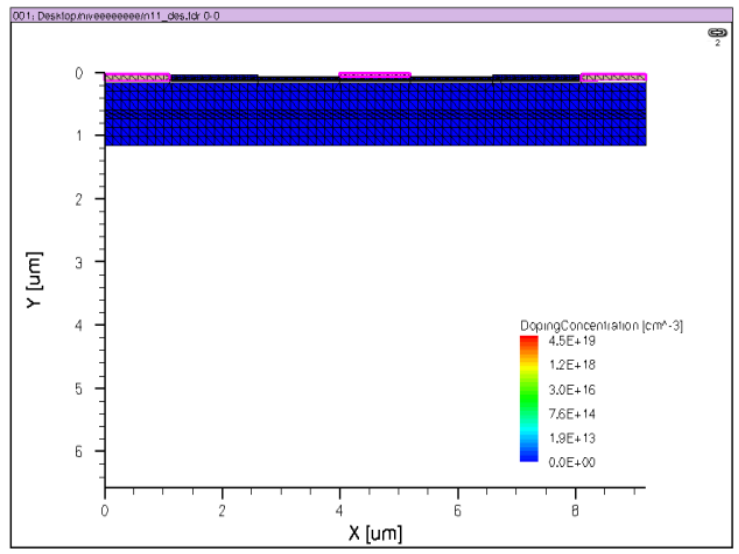

Figure.1 Meshing Structure of AIN InN\AIGaN MOS-HEMTs

\section{OVERVIEW OF TCAD TOOLS}

The Technology Computer Aided Design is a technology that resolves the equations on behalf of the manufacturing process of transistors in larger scale or equations in lieu of the physical/chemical phenomena related to electrical conduction. TCAD simulates and forecasts characteristics of a transistor or semiconductor circuit and thus surges the efficiency in planning and expansion of LSI [2].

Technology CAD is an outlet of electronic design automation model of semiconductor under fabrication and the device operation. It including of the modeling of development phases of diffusion and ion implantation and exhibiting the performance of the electrical devices based on essential physics, for instance the doping outlines of the devices. TCAD may also comprise the creation of compact models which attempt to internment the electrical performance of such devices which not fall underlying these devices physics.

\section{RESULTS AND DISCUSSION}

The most widely used gate dielectrics is $\mathrm{SiO}_{2}$ because of its natureof the higher barrier value between conduction bands of $\mathrm{GaN}$ and dielectric layer[6].In this work we given a deeper insight for investigation of dielectric materials directed on high-k dielectric constant value of the materials aluminum oxide $\left(\mathrm{Al}_{2} \mathrm{O}_{3}\right)[8]$ and hafnium oxide $\left(\mathrm{HfO}_{2}\right)$ [9].The response of device performance for varying barrier layer and high 2DEG density, a high drain current density and high trans conductance are verified. Further the device performance of DC transfer characteristics, small signal analysis of AlN/InN/AlGaNMOS-HEMT are analyzed and simulated.

The model is validated by change in composite material of $\mathrm{AlN} / \mathrm{InN} / \mathrm{AlGaN}$ with the respected to mole fraction $\mathrm{Al}_{0.30} \mathrm{GaN}_{0.70}$, then meshing of the device channel material has resulted in altered basics performance of the device in DC and RF characteristics. By means of changing the dielectric constant forSiO${ }_{2}$ toHfO $\mathrm{H}_{2}$ i.e., range between the $3.9-15$, these metric value deviation duly held for modification of the on-state current value [10].The employing of $\left(\mathrm{Al}_{2} \mathrm{O}_{3}\right) /\left(\mathrm{HfO}_{2}\right)$ dielectric layers with a high dielectric constant leads to superior conductivity in the device channel and it usually result in upsurge of the drain current owing to the on-state trans conductance for power applications.

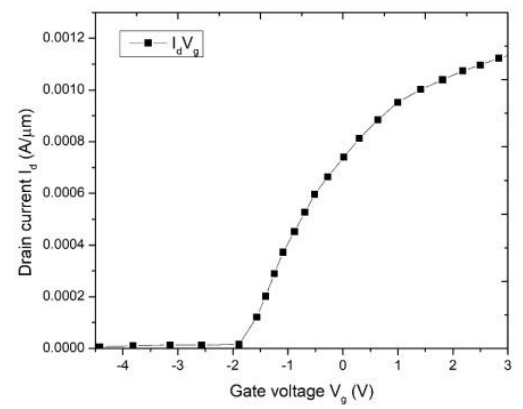

Figure.2 Drain current Vs Gate voltage characteristics of AINIInN\AIGaN HEMT

Figure 2demonstrations the variations of gate voltage $\left(\mathrm{V}_{\mathrm{g}}\right)$ with respect to drain current $\left(\mathrm{I}_{\mathrm{d}}\right)$.The maximum value of drain current $I_{d}$ obtained is around $0.0011(\mathrm{~A} / \mu \mathrm{m})$ for changes in the mole fraction of barrier interface layer $\mathrm{Al}_{0.30} \mathrm{GaN}_{0.70}$ and dielectric layer. These convention of high-K dielectrics leads to decreases in buildupof charge at the gate dielectric material $\mathrm{HfO}_{2}$, which in turn duly leads to reduced gate leakage current because of the quantum effects felt in the channel and limit the electrical characteristics variation for the given scaled devices[10].

Figure 3 shows the transfer characteristics of drain current $\left(\mathrm{I}_{\mathrm{d}}\right)$ vs. the drain voltage $\left(\mathrm{V}_{\mathrm{d}}\right)$. The gate voltage $\left(\mathrm{V}_{\mathrm{g}}\right)$ remains constant for value around -0.8 to $0.8 \mathrm{~V}$. The drain voltage is sweep up to $2.0 \mathrm{~V}$. The current saturate at $10 \mathrm{~V}$ and the maximum value of the drain current $0.0011(\mathrm{~A} / \mu \mathrm{m})$ is resulted with respected to change in the physical dimensions. The immobile charger existence are considerably note dat the lower energy band than at electron presence mobility charge at two-dimensional electron gasat channel, owing to their higher density at the interface states of gate dielectric material $\mathrm{HfO}_{2}$ and $\mathrm{GaN}$ layer. Furthermore, these carriers are able to scattered, due to the boundary roughness in the barrier layer of $\mathrm{Al}_{0.30} \mathrm{GaN}_{0.70}$ [10].



Figure 3. Drain current Vs drain voltage characteristics of AINIInNIAIGaN HEMT

Figure 4 shows the characteristics of Capacitance with respect to gate voltage of AlN $I n N \backslash A 1 G a N$ HEMT. The capacitance and gate voltage starts increasing linearly and becomes saturated at $10 \mathrm{~V}$ gate voltage.

\section{Published By}

Blue Eyes Intelligence Engineering \& Sciences Publication 
Hence the trap charge at the states of interface between the AlN $\backslash \mathrm{InN} \backslash \mathrm{Al}_{0.30} \mathrm{GaN}_{0.70}$ barrier layer and $\mathrm{HfO}_{2}$ oxide layer are investigate by variable applied bias in the depletion and accumulation region due to the dynamic capacitance dispersion technique [11] for improvement in the gate voltage and capacitance influences.

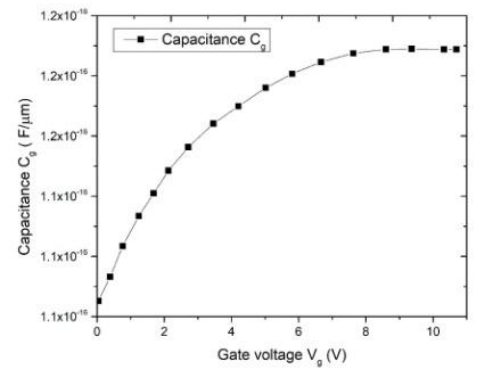

Figure 4. Capacitance Vs Gate Voltage Characteristics of AINIInN\AIGaN HEMT.

Figure 5 shows the trans conductance $(\mathrm{gm})$ variation with respect to voltage applied gate voltage. The value of the dielectric material of $\mathrm{HfO}_{2}$ interface with the barrier $\mathrm{Al}_{0.30} \mathrm{GaN}_{0.70}$ have been augmented for the improvement of 2DEG carrier concentration and its charge mobility. Henceforth by confirm low on-state resistance, vital for obtaining the higher charge carrier mobility in the region of conductive channel. The mobility resultant should be minimum during for normally-off MOS HEMTs With above related factor helps in the improvement in the trans conductance value around $0.0004(\mathrm{~S} / \mu \mathrm{m})$ for the device.



Figure 5.Transconductance $\left(g_{m}\right)$ Vs Gate voltageCharacteristics of AINIInNIAIGaN HEMT

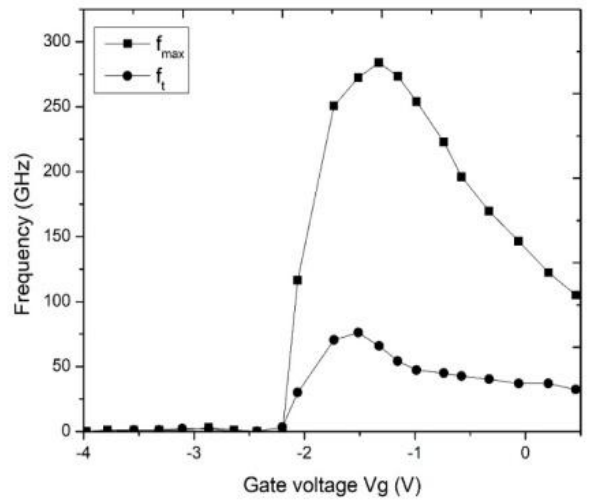

6. Frequency Vs Gate Voltage Characteristics of AINIInN\AIGaN HEMT
Figure 6. Shows a plots the $\mathrm{f}_{\mathrm{T}}$ and $\mathrm{f}_{\max }$ as a function of gate voltage for AlN\InN\AlGaNHEMT. The maximum value of $\mathrm{f}_{\mathrm{T}}$ andf $\mathrm{max}_{\text {max }}$ results around $275 \mathrm{GHz}$ and $75 \mathrm{GHz}$ respectively for

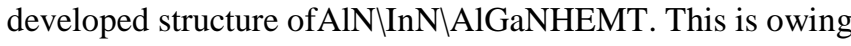
to improved output conductance characteristics of the because of the capacitance effects and mobility. Hence these device parameter plays a vital role in the field of low power and Radio frequency application regime.

\section{CONCLUSION}

The structure of the AIN\InN\AlGaN HEMT MOS-HEMTs are designed and meshed using the script file in the Sentaurus structure editor. AlN\InN\AlGaN MOS-HEMTs output characteristic are examined forphysics of MOS-HEMT in Hydrodynamic model TCAD. The process parameters corresponding mobility, band gap, and permittivity by means of based varying materials are created in parameter file for device simulation and those are validated for the response device structure in Senators TCAD using script and validated for meshrefinement for the analysis. Device performance like DC transfer characteristics, small signal analysis of MOS-HEMT are analyzed and simulated, the response of device performance for various device dimensions are compared. The amalgamation of barrier $\mathrm{Al}_{0.30} \mathrm{GaN}_{0.70}$ layer and formation virtual high $2 \mathrm{DEG}$ density results in higher drain current $\mathrm{I}_{\mathrm{d}}$ nearly $0.0011 \mathrm{~A} / \mu \mathrm{m}$, along with the high Trans conductance above $0.0004 \mathrm{~S} / \mu \mathrm{m}$, is achieved. The gate bias has been range between -0.8 to $0.8 \mathrm{~V}$ results in the maximum current density is high as $0.0011 \mathrm{~A} / \mu \mathrm{m}$ at $\mathrm{V}_{\mathrm{g}}=$ $0.08 \mathrm{~V}$, subsequent in an ON Resistance $\mathrm{R}_{\mathrm{ON}}$ of $0.185 \Omega$. At $\mathrm{V}_{\mathrm{d}}=10 \mathrm{~V}$, and maximum cut off frequency $\mathrm{ft}=75 \mathrm{GHz}$ and $\mathrm{f}_{\text {max }}=275 \mathrm{GHz}$.

\section{REFERENCES}

1. Ching-Sung Lee, Yu-Hao Liao, Bo-Yi Chou, Han-Yin Liu, Wei-Chou Hsu. "Composite HfO2/A12O3-dielectric AlGaAs/InGaAs MOSHEMTs by using RF sputtering/ozone water oxidation", Superlattices and Microstructures, 2014

2. M. Sheerin Begum, J. Vijayashree, A. Mohanbabu, N. Mohankumar. "Investigation of performance of InAsSb based high electron mobility transistors (HEMTs)", 2017 Devices for Integrated Circuit (DevIC), 2017

3. Lee, Ching-Sung, Yu-Hao Liao, Bo-Yi Chou, Han-Yin Liu, and Wei-Chou Hsu. "Composite HfO2/A12O3-dielectric AlGaAs/InGaAs MOS HEMTs by using RF sputtering/ozone water oxidation", Superlattices and Microstructures, 2014.

4. F. Medjdoub, J. Derluyn, K. Cheng, M. Leys, S. Degroote, D. Marcon, D. Visalli, M. Van Hove, M. Germain, G. Borghs. "Low On-Resistance High-Breakdown Normally Off AlN/GaN/AlGaN DHFET on Si Substrate", IEEE Electron Device Letters, 2010

5. Igor E. Anokhin, Matthias Wurl, Katia Parodi, Anatoly B. Rosenfeld "Application of silicon planar structures for the spectrum determination of the proton beams produced by laser-driven particle accelerators. Theoretical approach.", 2017 IEEE Nuclear Science Symposium and Medical Imaging Conference (NSS/MIC), 2017

6. Y. Niiyama et al.: Normally off operation GaN-based MOSFETs for power electronics applications, Semicond. Sci. Technol., 25, 125006, (2010).

7. T.E. Cook Jr. et al.: Measurement of the band offsets of $\mathrm{SiO} 2$ on clean n- and p-type GaN(0001), J. Appl. Phys. 93, 3995, (2003).

8. J.J. Freedsman et al.: Normally-off A12O3/AlGaN/GaN MOS-HEMT on $8 \mathrm{in}$. Si with low leakage current and high breakdown voltage (825 V), Appl. Phys. Express, 7, 04100, (2014).

9. W. Ahn et al.: Normally-off AlGaN/GaN MOS-HEMTs by KOH wet etch and RF-sputtered HfO2 gate insulator, Proceedings of 25th Int. Symp. on Power Semiconductor Devices \& IC's (ISPSD), pp.311-314, (2013).

Published By: 
10. Andrzej Taube, MariuszSochacki, Jan Szmidt, Eliana Kaminska, and Anna Piotrowska ,Modelling and Simulation of Normally-Off $\mathrm{AlGaN} / \mathrm{GaN}$ MOS-HEMTs Intl Journal Of Electronics and Telecommunications, 2014, VOL. 60, NO. 3, PP. 253-258 2014. DOI: 10.2478/eletel-2014-0032.

11. X.-H. Ma, J.-J. Zhu, X.-Y. Liao, T. Yue, W.-W. Chen, and Y. Hao, "Quantitative characterization of interface traps in Al2O3/AlGaN/GaN metal-oxide-semiconductor high- electron mobility transistors by dynamic capacitance dispersion technique," Applied Physics Letters, vol. 103, p. 033510, 2013. 